University of Wollongong

Research Online

Faculty of Engineering and Information

Faculty of Engineering and Information

Sciences - Papers: Part A

Sciences

$1-1-2012$

\title{
Rietveld analysis of the effect of annealing atmosphere on phase evolution of nanocrystalline TiO 2 powders
}

\author{
M Salari \\ University of Wollongong, msalari@uow.edu.au \\ M Rezaee \\ University of Wollongong \\ A T. Chidembo \\ University of Wollongong, atc987@uowmail.edu.au \\ Konstantin Konstantinov \\ University of Wollongong, konstan@uow.edu.au \\ Hua-Kun Liu \\ University of Wollongong, hua@uow.edu.au
}

Follow this and additional works at: https://ro.uow.edu.au/eispapers

Part of the Engineering Commons, and the Science and Technology Studies Commons

Research Online is the open access institutional repository for the University of Wollongong. For further information contact the UOW Library: research-pubs@uow.edu.au 


\title{
Rietveld analysis of the effect of annealing atmosphere on phase evolution of nanocrystalline TiO 2 powders
}

\author{
Abstract \\ The struclUral evolution of nanocrystaUine Ti02 was studied by X-ray diffraction (XRD) and the Rietveld \\ refinement method (RRM). Ti02 powders were prepared by the sol-gel technique. Post annealing of \\ as synthesized powders in the temperature range from $500^{\circ} \mathrm{C}$ to $600^{\circ} \mathrm{C}$ under air and argon atmospheres \\ led to the formation of Ti02 nanoparticles with mean crystallite size in the range of $37-165 \mathrm{~nm}$. based on \\ the Rietveld refinement results. It was found that the phase structure, composition, and crystallite size of \\ the resulting particles were dependent on not only the annealing temperature, but also the annealing \\ atmosphere. Rietveld refinement of the XRD data showed that annealing the powders under argon \\ atmosphere promoted the polymorphic phase transformation from anatase to rutile. Field emission \\ scanning electron microscopy (FESEM) was employed to investigate the morphology and size of the \\ annealed powders.

\section{Keywords} \\ rietveld, powders, 2, tio, analysis, nanocrystalline, effect, evolution, phase, atmosphere, annealing

\section{Disciplines} \\ Engineering | Science and Technology Studies

\section{Publication Details} \\ Salari, M., Rezaee, M., Chidembo, A. T., Konstantinov, K. \& Liu, H. K. (2012). Rietveld analysis of the effect \\ of annealing atmosphere on phase evolution of nanocrystalline TiO 2 powders. Journal of Nanoscience \\ and Nanotechnology, 12 (6), 4724-4728.
}




\title{
Rietveld Analysis of the Effect of Annealing Atmosphere on Phase Evolution of Nanocrystalline $\mathrm{TiO}_{2}$ Powders
}

\author{
M. Salari ${ }^{1}$, M. Rezaee ${ }^{1,2, *}$, A. T. Chidembo ${ }^{1}$, K. Konstantinov ${ }^{1}$, and H. K. Liu ${ }^{1}$ \\ 1 Institute for Superconducting and Electronic Materials (ISEM), ARC Centre for Electromaterials Science, \\ University of Wollongong, NSW 2500, Australia \\ ${ }^{2}$ Mining and Metallurgical Engineering Department, Amirkbair University of Technology, \\ Tehran, Iran, P.O. Box 158754413
}

\begin{abstract}
The structural evolution of nanocrystalline $\mathrm{TiO}_{2}$ was studied by X-ray diffraction (XRD) and the Rietveld refinement method (RRM). $\mathrm{TiO}_{2}$ powders were prepared by the sol-gel technique. Post annealing of as-synthesized powders in the temperature range from $500^{\circ} \mathrm{C}$ to $800^{\circ} \mathrm{C}$ under air and argon atmospheres led to the formation of $\mathrm{TiO}_{2}$ nanoparticles with mean crystallite size in the range of $37-165 \mathrm{~nm}$, based on the Rietveld refinement results. It was found that the phase structure, composition, and crystallite size of the resulting particles were dependent on not only the annealing temperature, but also the annealing atmosphere. Rietveld refinement of the XRD data showed that annealing the powders under argon atmosphere promoted the polymorphic phase transformation from anatase to rutile. Field emission scanning electron microscopy (FESEM) was employed to investigate the morphology and size of the annealed powders.
\end{abstract}

Keywords: Rietveld Refinement Method, Sol-Gel, $\mathrm{TiO}_{2}$, Phase Transformation, Annealing Atmosphere.

\section{INTRODUCTION}

The physical and chemical properties of nanocrystalline materials often differ from those of crystalline or amorphous ones. Titanium dioxide is a material which has been widely investigated, and due to its wide range of applications in photocatalysis, pigments, protection against ultraviolet irradiation, ${ }^{1-5}$ sensors, ${ }^{6}$ antibiosis, ${ }^{5}$ and so on, it has been of considerable interest in recent years. However, a large amount of work needs be done to realize its full potential. The effective introduction of nanocrystalline particles in the applications listed above requires careful investigation of the crystallite size of these particles to make the most of their exceptional properties. ${ }^{1}{ }^{6.7}$ Almost all results show that the smaller the particle size, the better the various properties, revealing the superior performance of nanocrystalline titania. When heated, nanocrystalline anatase will coarsen and be transformed to rutile, which will continue to coarsen much faster. ${ }^{8}$

The ease of data collection in powder diffraction instruments, and their availability and versatility make them indispensable tools to obtain compositional, structural,

-Author to whom correspondence should be addressed. microstructural, and many other types of information. The extensive peak broadening and possible overlapping inherent in studies of the patterns acquired from nanoparticles make the phase determination process a very demanding and time-consuming one. Misidentification of multiple phase systems, co-existing in closely related phases, as being single phase ones can lead to deceptive results. ${ }^{9}$ Therefore, full-pattern profile fitting techniques are extensively employed to assist in the phase identification process for quantification purposes. Among the various established methods for the analysis of X-ray diffraction (XRD) patterns, the Warren-Averbach ${ }^{10.11}$ method is highly regarded as an elaborate and rigorous method for size-strain analysis in order to evaluate microstructural parameters such as domain size $(\langle D\rangle)$, micro-strain $\left(\left\langle\varepsilon^{2}\right\rangle^{1 / 2}\right)$, etc. In this approach, the domain size and microstrain can be assessed by expressing the observed XRD profile in the form of a Fourier series. The coefficients used in this method are related to the effects of deformations and instrumental effects. The instrumental effects can be determined by implementing Stoke's method (i.e., by deconvoluting the Fourier coefficients of the Fourier series into those obtained from the profile of standard silicon). ${ }^{12}$ Another approach is the Rietveld ${ }^{13}$ method (a full-pattern 
fit method). The Rietveld method is a well-known powderpattern fitting method, which consists of adjusting a theoretical diffraction pattern, calculated on the basis of a model of the crystal structure of the sample, with experimental recorded data. The method is most applicable to XRD data and to synchrotron and neutron diffraction data. There has also been a trend to apply this method to electron powder diffraction pattern data. ${ }^{4.14}$

The Rietveld method exhibits advantages over all the other methods. It takes into account the whole fitting methodology for size-strain analysis, simultaneous refinement of the crystal structure, and microstructural parameters, and is capable of eliminating preferred orientation effects (if there are any). ${ }^{12,15}$

Considering all the benefits of the whole profile fitting methodology, the Rietveld structure refinement procedure using MAUD software has been adopted in the present study to address the effect of the annealing temperature and atmosphere as the operative parameters on the phase structure, phase composition, and crystallite size of nanocrystalline $\mathrm{TiO}_{2}$ powders prepared by the sol-gel method.

\section{EXPERIMENTAL PROCEDURE}

The samples of nanocrystalline $\mathrm{TiO}_{2}$ were synthesized by two-stage hydrolysis of titanium (IV)-isopropoxide (TTIP, Aldrich). TTIP was first diluted with absolute ethanol (TTIP/Ethanol $=1 / 5)$ while stirring. De-ionized water (TTIP/ $\mathrm{H}_{2} \mathrm{O}=1 / 20$ ) was then added into the diluted solution together with acidic $(\mathrm{HCl})$ and basic $\left(\mathrm{NH}_{4} \mathrm{OH}\right)$ catalytic agents. The solution was stirred at room temperature for 1 hour. The final white slurry was filtered and dried at $100^{\circ} \mathrm{C}$ overnight. In the present work, four sets of experiments with different annealing atmospheres and annealing temperatures were performed (Table I).

Phase characterization of the obtained powder was conducted using GBC MMA X-Ray diffraction (XRD) with $\mathrm{Cu}-\mathrm{K}_{\alpha}$ radiation. The average crystallite size and relative weight percentage of the phases in the samples were calculated via the Rietveld refinement method using MAUD software. The particle size and morphology were investigated using a JEOL JSM-7500FA field emission scanning electron microscope (FESEM).

Table I. Annealing conditions of the as-synthesized samples.

\begin{tabular}{lcc}
\hline Sample & $\begin{array}{c}\text { Annealing temperature } \\
\left({ }^{\circ} \mathrm{C}\right)\end{array}$ & $\begin{array}{c}\text { Annealing atmosphere } \\
\text { A }\end{array}$ \\
& 500 & Air \\
& & Argon \\
B & 600 & Air \\
& & Argon \\
C & 700 & Air \\
& & Argon \\
D & 800 & Air \\
& & Argon \\
\hline
\end{tabular}

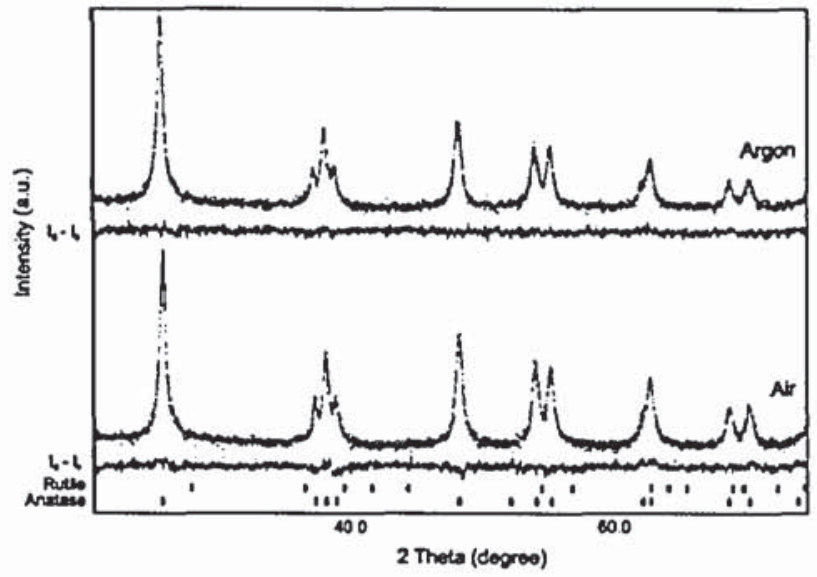

Fig. 1. XRD panterns of samples annealed at $500^{\circ} \mathrm{C}$ (set $\mathrm{A}$ ) under air and argon atmospheres.

\section{RESULTS AND DISCUSSION}

In order to determine the exact phase evolution in the $\mathrm{TiO}_{2}$ powders, it is essential to fit the experimental powder diffraction pattern with a profile fitting function based on the crystal structure and microstructure information. This methodology takes care of all reflections of each phase present in such a multiphase material and provides a very accurate result. The experimental XRD pattern (dots) of the sample annealed at $500{ }^{\circ} \mathrm{C}$ (set A), fitted with its calculated counterpart (solid curve), is shown in Figure 1. The differences between the experimental and the fitted patterns shown as the continuous line under the individual diffraction pattern. The vertical bars at the bottom of the figure indicate the diffraction peak positions of different $\mathrm{TiO}_{2}$ phases. This figure reveals that the samples completely consist of anatase phase.

The experimental XRD patterns (dots) of the samples annealed at $600{ }^{\circ} \mathrm{C}$ (set B), fitted by the theoretical patterns calculated based on their crystallographic file information (CIF), is presented in Figure 2. From the plots

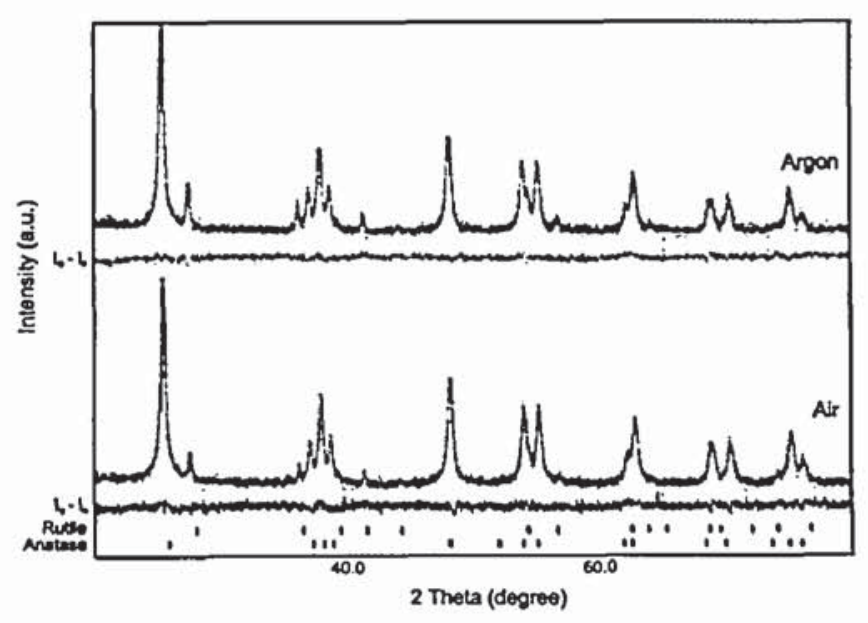

Fig. 2. XRD patterns of samples annealed at $600^{\circ} \mathrm{C}$ (set B) under air and argon atmospheres. 


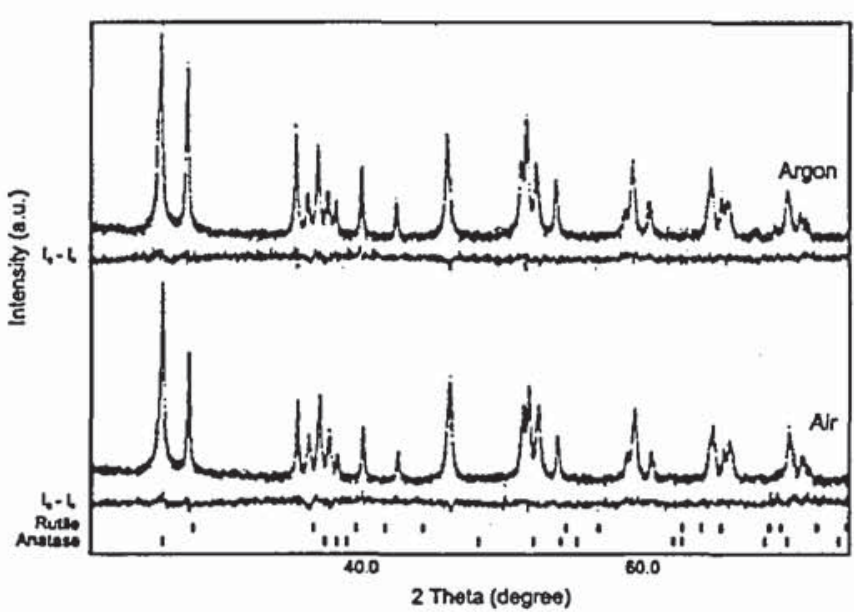

Fig. 3. XRD patterns of samples annealed at $700^{\circ} \mathrm{C}$ (sample C) under air and argon atmospheres.

of the differences between the experimental and the fitted patterns, it is clearly evident that the experimental patterns have been fitted satisfactorily. It can be observed that these samples consist of anatase phase and a small hint of rutile phase. In fact, annealing at higher temperature caused the rutile peaks to appear. This can be attributed to the coarsening of the initial anatase grains with increasing annealing temperature and subsequent coarsening of the final anatase grains. ${ }^{16}$

As can be seen in Figure 2, the intensity ratio of the rutile peak to the anatase one is higher for the sample annealed under argon atmosphere than for the one annealed in air atmosphere. Since the anatase to rutile phase transformation involves an overall contraction of the oxygen structure and a cooperative movement of ions, the removal of oxygen ions might be expected to accelerate the transformation, whereas the formation of titanium interstitials would inhibit the transformation. This implies that an argon atmosphere would accelerate the transformation. Conversely, the presence of interstitials would increase the strain energy that would have to be overcome and the

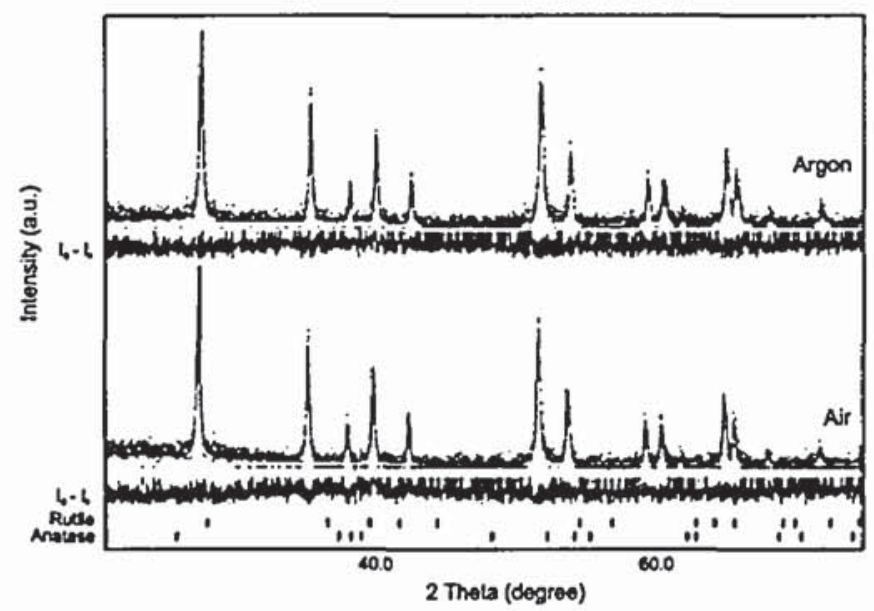

Fig. 4. XRD pattems of samples annealed at $800^{\circ} \mathrm{C}$ (sample D) under air and argon atmospheręs.

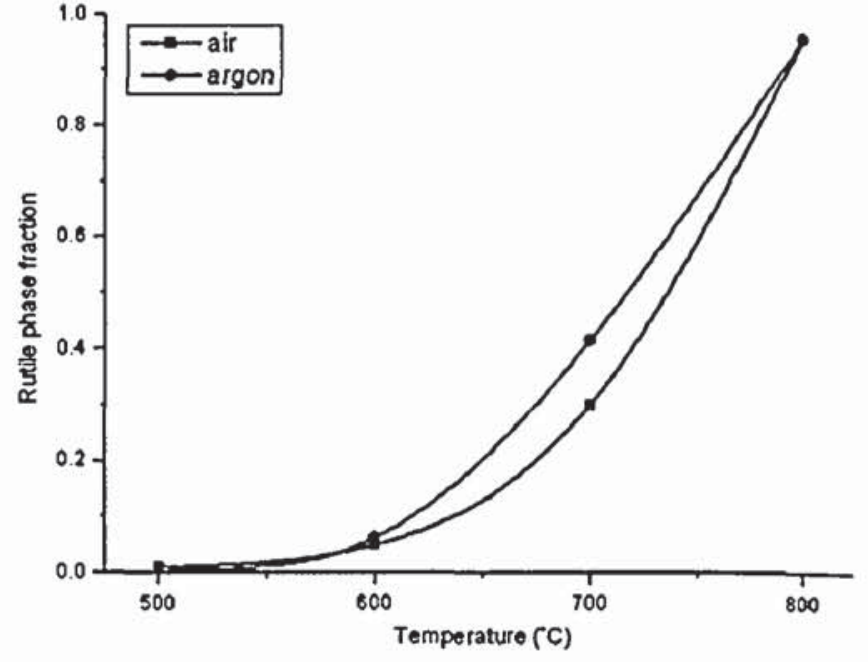

Fig. 5. Rutile phase fraction with increasing annealing temperature.

bonds that would have to be broken, implying that an air atmosphere would inhibit the transformation. ${ }^{17,18}$

This trend towards increase of the polymorphic transformation with increasing annealing temperature and change of the annealing atmosphere from air to argon can be observed by annealing the samples at $700{ }^{\circ} \mathrm{C}$ (Set C). Figure 3 presents the experimental XRD patterns (dots) of samples annealed at $700{ }^{\circ} \mathrm{C}$ fitted with the corresponding simulated patterns (solid line). As can be seen in this figure, both samples are a mixture of anatase and rutile phases. It is obvious that the relative content of rutile phase in this set is higher than in sets A and B.

Annealing the samples at the higher temperature of $800^{\circ} \mathrm{C}$ (set $\mathrm{D}$ ) completes the anatase to rutile phase transformation in both atmospheres. Figure 4 shows the experimental XRD patterns (dots) of these samples fitted with their theoretical counterparts (solid lines).

Figure 4 shows that the polymorphic transformation can be completed in all the samples by annealing them

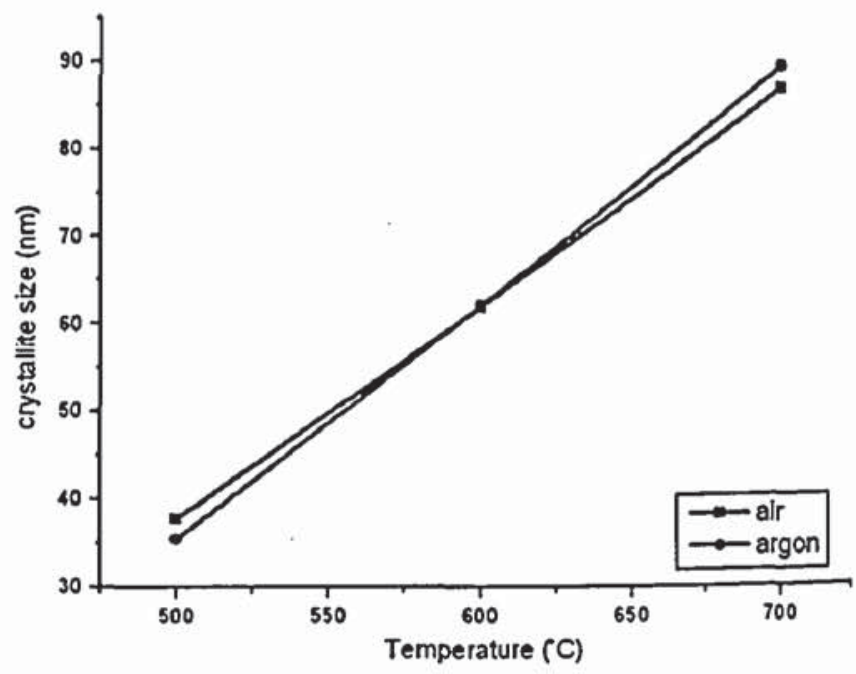

Fig. 6. Mean crystallite size of anatase phase versus annealing temperature, as calculated by the Rietveld refinement method. 

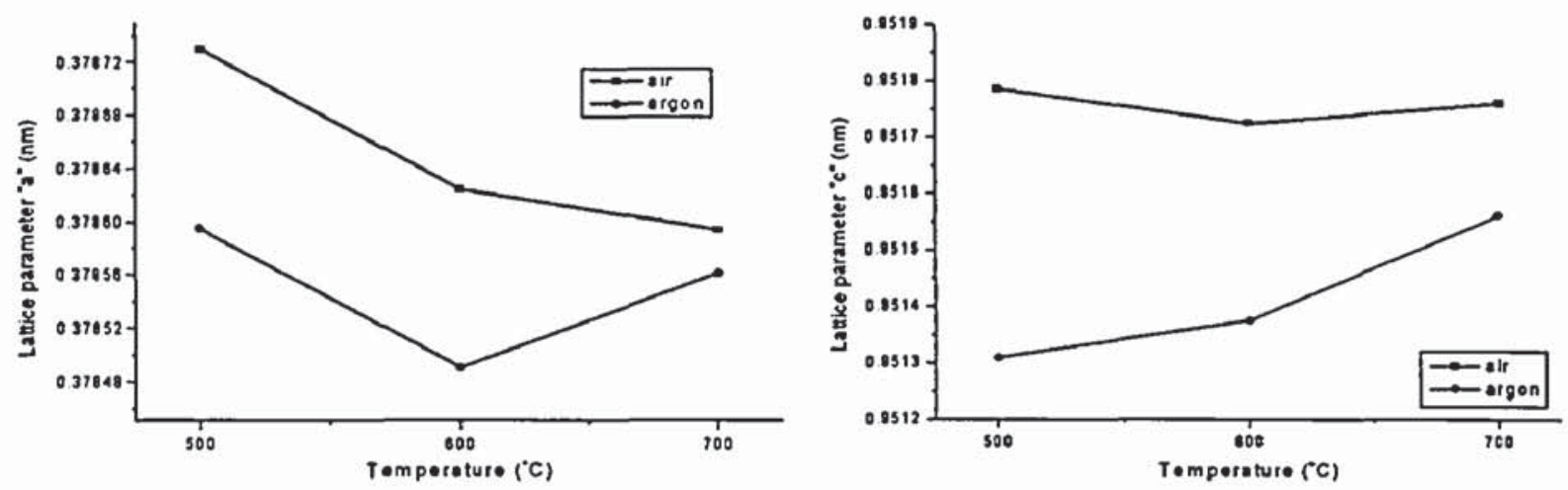

Fig. 7. " $\mathrm{a}$ " and " $\mathrm{c}$ " lattice parameters of anatase phase for the samples annealed under air and argon atmospheres at $500^{\circ} \mathrm{C}, 600^{\circ} \mathrm{C}$, and $700{ }^{\circ} \mathrm{C}$.

at $800{ }^{\circ} \mathrm{C}$, whereas at lower annealing temperatures, the transformation rate in the samples annealed under argon atmosphere is higher than for the samples annealed in air.

Figure 5 shows the variation in the rutile phase fraction after the anatase to rutile phase transformation, calculated using the Rietveld refinement method in the samples annealed in air and under argon atmosphere, with annealing at different temperatures for a period of 1 hour. It can be observed that the phase transformation progression in both samples obeys a sigmoidal shape and that the rate of the phase transformation in the powders annealed under argon atmosphere is higher than in the samples annealed in air.

Kinetic considerations suggest that the oxygen partial pressure of the annealing atmosphere may have an influence on the concentration of oxygen vacancies in the $\mathrm{TiO}_{2}$ lattice. An annealing atmosphere with low oxygen partial pressure favors the formation of oxygen vacancies and consequently accelerates the phase transformation of anatase. ${ }^{5}$ Thus, in the nanocrystalline $\mathrm{TiO}_{2}$ powders annealed in air and argon atmospheres, the transformation rates of anatase are different, although the temperatures experienced by the powders are the same. More oxygen vacancies form in the argon-annealed $\mathrm{TiO}_{2}$ powder due to lower oxygen partial pressure, which enhances the transformation of anatase.

Figure 6 shows the change in anatase crystallite size with increasing annealing temperature in samples annealed under argon and in air atmospheres. This figure demonstrates that the mean crystallite size of the anatase phase increases with increasing annealing temperature in both samples.

As a rule, nanostructured materials contain a large number of disordered surface atoms which possess greater energy than the inner atoms. As the size of a grain becomes smaller, its surface area increases, and the surface atoms exhibit higher mobility, which leads to greater defect content, thus further enlarging lattice deformation. Previous studies have shown that nanocrystalline $\mathrm{TiO}_{2}$ is typically oxygen-deficient. ${ }^{8}$ Figure 7 shows the calculated lattice parameters of anatase phase in the samples annealed under air and argon atmospheres at the temperatures of $500^{\circ} \mathrm{C}, 600^{\circ} \mathrm{C}$, and $700^{\circ} \mathrm{C}$. As can be seen, the $a$ and $c$ parameters in all samples are different from the theoretical lattice parameters of anatase phase $\left(a_{0}=3.7852 \AA\right.$ and $c_{0}=9.5139 \AA$ ). The transformation from anatase to rutile during annealing seems to be a reasonable outcome due to the existing deformation in the powders.

Figure 8 shows two FESEM micrographs of the annealed samples. As can be seen, the sample annealed at $500^{\circ} \mathrm{C}$ (Fig. 8(a)), consisting of fully anatase phase, has smaller particles than the sample annealed at $800^{\circ} \mathrm{C}$ (Fig. 8(b)), consisting of fully rutile phase. The larger particle size

(a)

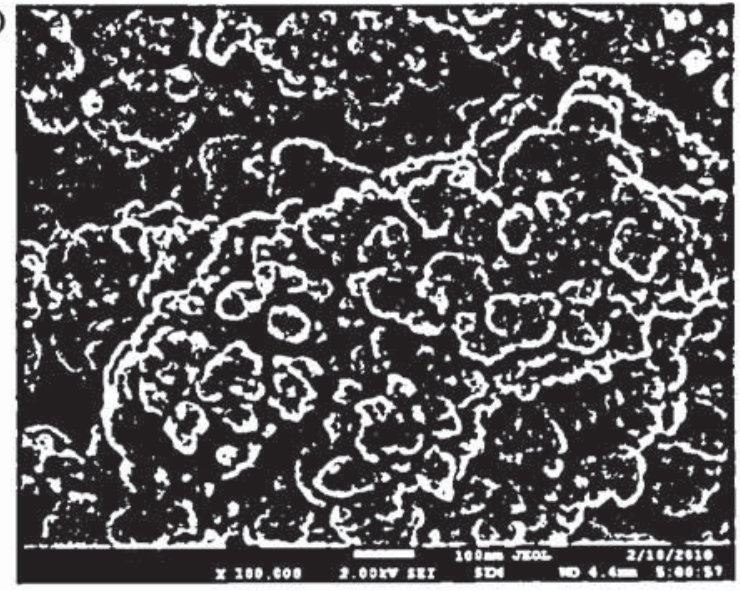

(b)

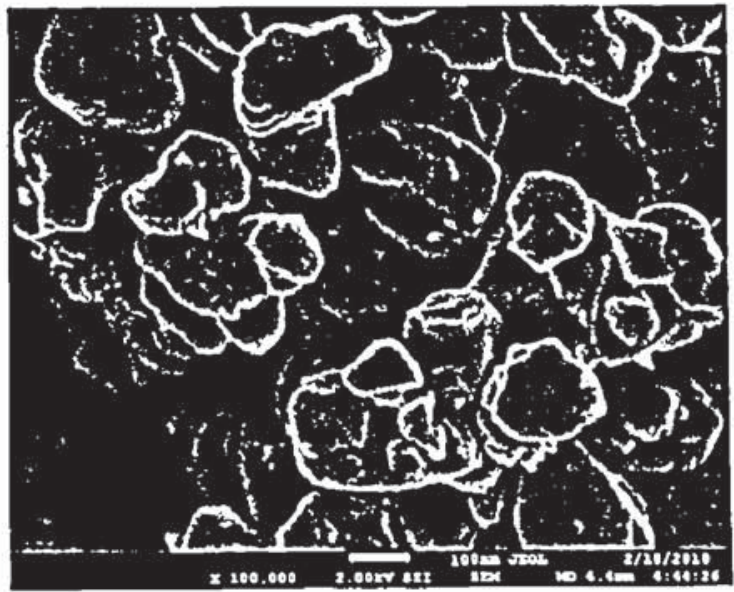

Fig. 8. FESEM micrographs of nanocrystalline $\mathrm{TiO}_{2}$ powders annealed (a) at $500^{\circ} \mathrm{C}$ in air and (b) at $800^{\circ} \mathrm{C}$ under argon atmosphere. 
in the sample annealed at $800{ }^{\circ} \mathrm{C}$ can be attributed to the higher annealing temperature. ${ }^{19}$ The other noticeable point is the fairly good agreement between the calculated crystallite size from the XRD patterns, based on the Rietveld refinement method, and the particle size in the FESEM micrograph for the sample annealed at $500{ }^{\circ} \mathrm{C}$, which means that each particle consists of just one crystallite. Because the size of the particles annealed at $800{ }^{\circ} \mathrm{C}$ is larger than the upper detection limit of $\mathrm{X}$-ray diffraction analysis based on the Rietveld method, the Rietveld method cannot be used to fully describe the pattern and therefore cannot be employed to determine the particle size. ${ }^{20}$ So, the calculated mean crystallite sizes of the rutile phase are not reported here.

\section{CONCLUSION}

The effects of the annealing temperature and the annealing atmosphere on the structural evolution of nanocrystalline $\mathrm{TiO}_{2}$ powders prepared by the sol-gel method were investigated. It was observed that the transformation of anatase to rutile in all samples began at $500^{\circ} \mathrm{C}$ and was completed at $800^{\circ} \mathrm{C}$, regardless of annealing atmosphere. However, the transformation rate of anatase to rutile was accelerated by annealing the powders under argon atmosphere due to the decreased oxygen partial pressure of the annealing atmosphere, which favors the formation of oxygen vacancies in the nanocrystalline $\mathrm{TiO}_{2}$ particles. A good agreement was found between the particle size in FESEM micrographs and the calculated results for crystallite size based on the Rietveld refinement method, which means that the particles are single crystallites.

Acknowledgments: Financial support from the University of Wollongong and the Australian Research Council
(ARC, grant no. DP1093952) is gratefully acknowledged. The authors would like to thank Dr Tania Silver for critical reading of the manuscript, and S. Hamed Aboutalebi for valuable discussions.

\section{References and Notes}

1. P. Cheng, M. Zheng, Y. Jin, Q. Huang, and M. Gu, Mater. Lett. 57, 2989 (2003).

2. S. R. Dhage, V. D. Choube, V. Samuel, and V. Ravi, Mater. Lett. $58,2310(2004)$.

3. J.-G. Li and T. Ishigaki, Acta Mater. 52, 5143 (2004).

4. A. M. Tonejc, I. Djerdj, and A. Tonejc, Mater. Sci. Eng., C 19,85 (2002).

5. X. Pan and X. Ma, J. Solid State Chem. 177, 4098 (2004).

6. G. L. Li and G. H. Wang, Nanostruct. Mater. 11, 663 (1999).

7. Y. Li, T. J. White, and S. H. Lim, J. Solid State Chem. 177, 1372 (2004).

8. Y. Shao, D. Tang, J. Sun, Y. Lee, and W. Xiong, China Particuology 2. 119 (2004).

9. P. Whitfield and L. Mitchell, International Journal of Nanoscience 3. 757 (2004).

10. B. E. Warren and B. L. Averbach, J. Appl. Phys. 21, 595 (1950).

11. B. E. Warren and B. L. Averbach, J. Appl. Phys. 23, 497 (1952).

12. J. Ghosh, S. Chattopadhyay, A. Meikap, S. Chatterjee, and P. Chatterjec, Bull. Mater. Sci. 30, 447 (2007).

13. H. Rietveld, J. Appl. Cnstallogr. 2, 65 (1969).

14. X. Y. Wang, Z. Liu, H, Liao, D. Klein, and C. Coddet, Thin Solid Films 473, 177 (2005)

15. J. Ghosh, S. K. Chattopadhayay, A. K. Meikap, and S. K. Chatterjee, J. Alloy's Compd. 453, 131 (2008).

16. M. Salari, M. Rezaec, S. M. Mousavi Koic, P. Marashi, and H. Aboutalebi, Int. J. Mod. Phys. B 22, 2955 (2008).

17. R. D. Shannon and J. A. Pask, J. Am. Ceram. Soc, 48, 391 (1965).

18. F. Lin, D. Jiang, Y. Lin, and X. Ma, Physica B: Condensed Matter 403, 2193 (2008).

19. M. Salari, S. M. Mousavi khoie, P. Marashi, and M. Rezaee, J. Alloys Compd. 469, 386 (2009).

20. B. D. Cullity and S. R. Stock, Elements of X-Ray Diffraction, 3rd edn., Prentice Hall (2001).

Received: 1 December 2010. Accepted: 1 May 2011. 